\title{
CASE REPORT: DISSEMINATED AND CHRONIC MUCOCUTANEOUS CANDIDIASIS CAUSED BY CANDIDA LUSITANEA
}

Leonardo G. Artoni ${ }^{1}$, Paulo V. Soeiro Pereira', Leônidas L. Braga Júnior ${ }^{1}$, Annie Mafra Oliveira ${ }^{1}$, Carla Regina F. Grosse da Silva', Lais

C. L. Alencar ${ }^{1}$, Matheus Cardoso Silva², Daniel Wagner C. L. Santos ${ }^{3}$, Conceição de M. P. S. Azevedo'. 1- UFMA, 2-CEUMA, 3UNISFESP.

\section{OBJECTIVE}

To describe a clinical case of disseminated mucocutaneous candidiasis caused by Candida

lusitanea in a 2 year old child with a late diagnosis.

\section{METHODS}

This is a clinical case description with an etiological diagnosis confirmed by MALDI-TOF® treated with amphotericin $\mathrm{B}$.

\section{RESULTS}

Male, 2 years old, $8 \mathrm{~kg}$, from Queimadas - Codó (Maranhão /Brazil), in October 2017. The patient was hospitalized at the Materno Infantil University Hospital (MIUH) of the Federal University of Maranhão, Brazil. Ever since aged 7 months, the child had papillary-erythematous, pruriginous lesions in the thorax and cervical region, which improved after 15 days with topical use of ketoconazole. He presented new episodes of exacerbations and remissions, until be admitted to a hospital in the countryside, in april, 2017, with pneumonia, and then transferred to the MIUH. At physical examination: Bad general condition, inadequate weight-for-length growth, erythematous, desquamative plaques, raised and well delimited borders on the face, trunk, upper limbs, genital region, thigh root, palmar hyperkeratosis (onirodesthrosis on quirodactyls ), crusty, dark, coalescing lesions on the face (fig 1). It also had whitish plaques in the oral cavity and oropharynx. Respiratory system with positive vesicular breathing, presenting snores bilaterally. Antecedents: Pneumonia at 45 days of age, adverse effect to BCG, incomplete vaccination portfolio, no history of chronic or genetic pathologies in the family. Laboratory tests: Hemogram: (1st) hemoglobin: $8.49 \mathrm{~g} / \mathrm{dL}$; hematocrit: $26.5 \%$; leukocytes: $13,000 \mathrm{p} / \mathrm{mm}^{3}$; neutrophils: 7,540 (58\%); direct mycological examination: numerous hyaline hyphae and arthroconidia; Sabouraud agar lesion scraping culture: Candida lusitanea (confirmed in MALDI-TOF), sensitive to fluconazole and micafungin; Anti-HIV serology: non-reactive. Treatment/Evolution: Treatment Initiated with azithromycin, maintained for 7 days, did not improve respiratory symptoms. Initiated fluconazole therapy (6mg / kg / day) VO, associated with topical mineral oil and ketoconazole. After 15 days of hospitalization, the general condition had improved, with the onset of remission of the crusted lesions and a slight improvement in the pulmonary condition. On the 17th day of hospitalization (13th day of fluconazole), druginduced hepatitis was diagnosed, and the treatment with fluconazole was interrupted. The patient was discharged after 26 days of hospitalization, with 90\% of lesions in remission, with a prescription of ketoconazole cream for outpatient follow-up.

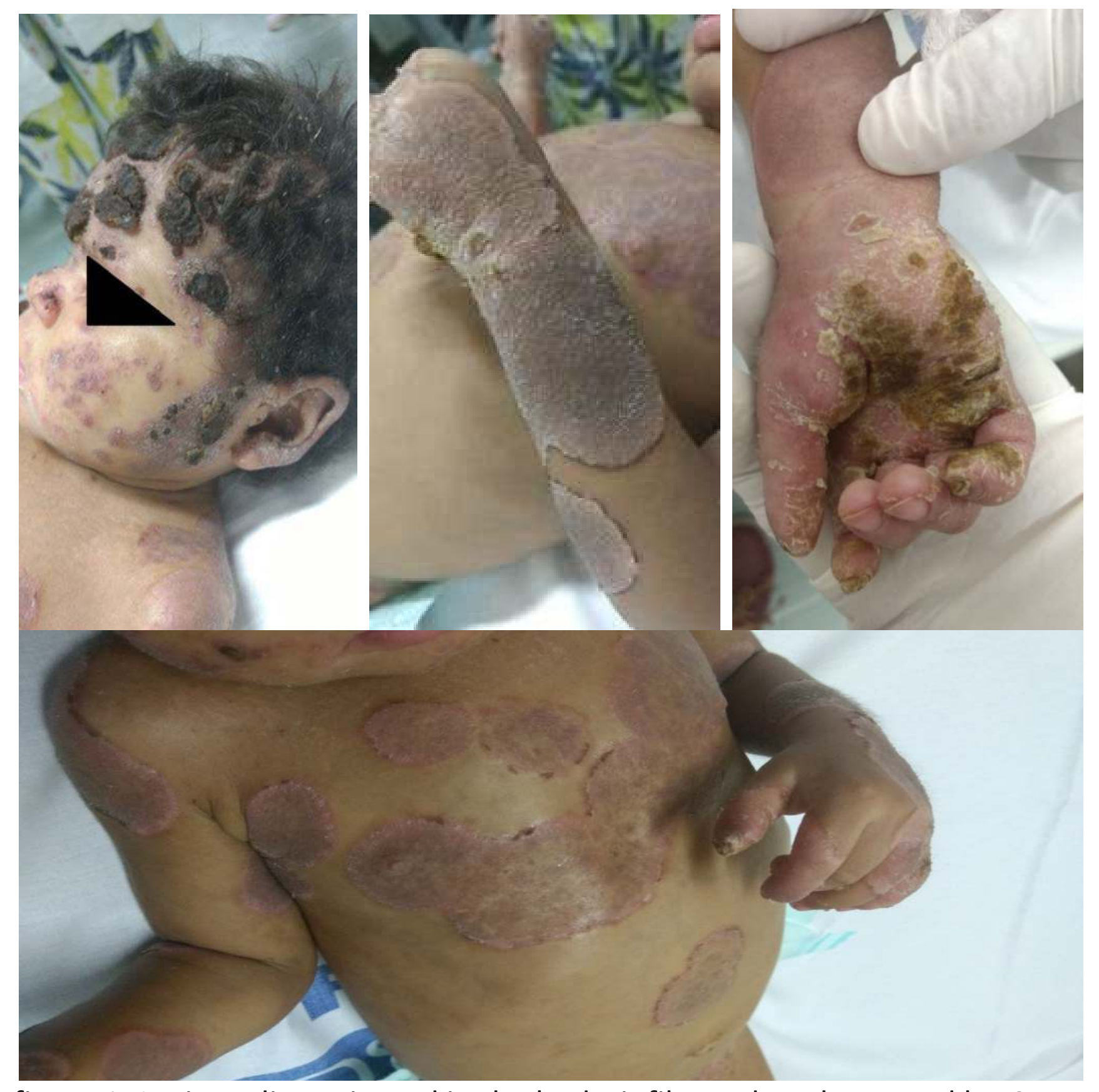

figure 1- Lesions disseminated in the body, infiltrated, scaly, caused by $C$. lusitaneae. Source: Author.

\section{CONCLUSION}

The case suggests a primary defect of innate immunity, probably in phagocytes (macrophages and neutrophils). However, further studies will be conducted to elucidate the etiology of the manifestations. 